\title{
Laboratory utilization in the emergency department - are the requested tests patient-oriented?
}

\author{
IVANA LAPIĆ, DUNJA ROGIĆ \\ Corresponding author: \\ Ivana Lapić \\ University Hospital Centre Zagreb \\ Kispatićeva 12, 10000 Zagreb \\ E-mail:ivana.lapic@hotmail.com
}

Department of Laboratory Diagnostics, University Hospital Centre Zagreb, Zagreb, Croatia

\section{ABSTRACT}

Emergency laboratory services are essential for the efficient management of critically ill patients admitted to the emergency department. Characterised by their high priority of processing, analysing and reporting, they serve as a discriminating tool in the triage of medical emergencies, rather than as a diagnostic tool. Optimal and adequate utilisation of laboratory testing greatly contributes to the overall quality and efficiency of laboratory services. From our experience gained through monitoring the ordering habits among physicians at the Emergency Medical Service of the University Hospital Centre Zagreb, nonselective overuse of laboratory tests occurs in practice. The most commonly used laboratory tests are often ordered together and at negligible variation between physicians, regardless of a widely varied patient population. Patient-oriented laboratory diagnostics can be achieved through limiting the availability of test ordering, an application of test panels based on relevant guidelines and protocols and upkeep of continuous close interaction between clinicians and laboratory experts.

Key words: emergency laboratory tests, laboratory utilization

\section{INTRODUCTION}

Laboratory tests are one of the fundamental tools used in making decisions in medical practice. Emergency laboratory tests form a unique subgroup of tests and services characterised by their high priority of processing, analysing and reporting. An efficient laboratory is considered to be one of the essential requirements for the effectiveness of the emergency department. Rapid, reliable and accurate laboratory reports should be provided within minutes to allow emergency department staff to manage medical emergencies. Rapid analytical turnaround time (TAT) is considered to be one of the most important quality indicators of the emergency laboratory. $(1,2)$ To both meet the objectives of the emergency department and contribute to its successful operation, emergency laboratories must apply various principles and procedures that set them apart them from the central laboratory. Reliable and optimal throughput analysers, a well-trained laboratory technician staff and a shift from a centralized to a decentralized laboratory located in close proximity to the emergency department allows continuous workflow and benefits high quality management of critically ill patients admitted to the emergency department. (1) However, optimal and adequate utilisation of laboratory testing greatly contributes to the overall quality and efficiency of laboratory services. Laboratory tests in the emergency department are primarily ordered to either confirm or refute the working diagnosis established after physical examination. Triage of a widely varied patient population admitted to the emergency department, rather than differential diagnostics, is the main purpose of laboratory testing in the emergency department. A rational panel of laboratory tests should be performed in order to provide appropriate patient care on the one hand, and to avoid unnecessary costs and delays in diagnosis on the other. $(1,3)$ At the University Hospital Centre Zagreb, the emergency laboratory for outpatients is an integrated part of the emergency department, allowing fast laboratory service. With the aim to rationalise requests, reduce inappropriate testing and costs, as well as to improve patient care, the ordering practices among physicians in the emergency department are being assessed on a regular basis. As a consequence, various educational and administrative in-

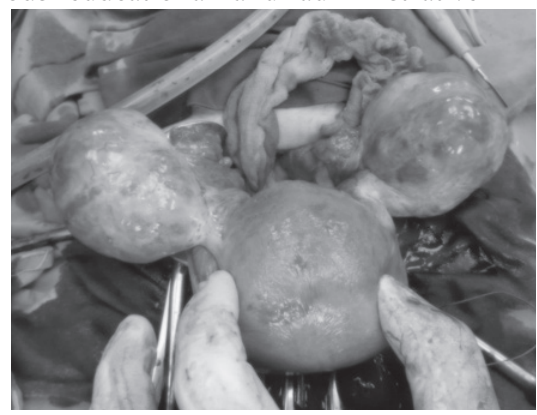

Figure 1. Intraoperative picture of the bilateral adnexal masses

terventions are being performed, and a continuous but steady effort is being made to improve communication between clinicians and the laboratory.

\section{WHERE ARE WE?}

Laboratory overuse is an ever growing phenomenon that most laboratories are nowadays facing. Increased workload and test diversity, as well as the widespread use of laboratory tests in the management of emergency patients, all contribute to a significant rise in laboratory expenses. (4) The roots of inappropriate test ordering behaviour derive from historical practice. It has long been accepted that the laboratory serves as a user-friendly service providing physicians with all information requested. The laboratory-physician relationship could be described as a vicious circle of test requests on one side, and test reports on the other. In an effort to provide the patient admitted to the emergency department with high-quality care, clinicians are unintentionally prone to excessive ordering of laboratory tests. This is mainly 
due to the possibility of ordering laboratory tests via multiple choice request papers, the use of settled protocols and an unawareness of the examination costs. Inappropriate initial testing can consequently deteriorate the quality of provided care by delays in diagnosis and misleading falsepositive results. Additionally, unnecessary costs are being generated. (4-6)

Today there is a necessity to bridge this gap. In our era of rationalisation, as well as continuous scientific and technological development, a shift from historical practice to best practice must be made. (4)

Patients admitted to the emergency department cover a wide range of symptoms and conditions, from the mild and harmless, to the life-threatening. Ordering the right tests at the right time (rather than the so-called "the more, the merrier" approach) may result in making the triage business in the emergency department extremely challenging, but it is essential in making the proper medical decision.

The emergency laboratory at the Emergency Medical Service of the University Hospital Centre Zagreb is a full-time and full-service equipped facility with biochemistry, immunochemistry, haematology and coagulation analysers providing laboratory diagnostics for outpatients. The panel of emergency laboratory tests can be divided into categories according to their priority in emergency states; acid-base status, glucose, haemoglobin and platelet count, electrolytes, prothrombin and activated thromboplastin time all form the first category of uppermost emergency tests crucial for the instant care of critically-ill patients. The group of secondary and tertiary laboratory tests includes organspecific examinations that contribute to a final diagnosis. (1) Laboratory test orders are processed via a request form in the Hospital Information System (HIS), they are consequently registered, and once the specimen has been delivered, the request is accepted by the laboratory staff through the Laboratory Information System (LIS). Automatic data exchange between LIS and automated analysers allows for the fast and reliable reporting of results and immediate access to laboratory data in HIS. Along with necessary tests, this system results in a number of potentially avoidable tests that are being requested simply because they are available.
Monitoring and tracking the ordering habits among physicians in the emergency department was a daunting task, but now, with massive amounts of data stored through LIS, the production of administrative reports of all kinds is easily feasible. We conducted a retrospective analysis of ordering practices among physicians in the emergency department over the course of a ten-month period (January - October 2014). In the observed period, an average of ten tests per patient was performed out of a total of 23,421 patients. A coefficient of variation of the relative number of all completed laboratory tests in a total number of patients per physician was less than $10 \%$. High-frequency testing was observed for emergency tests of the first priority. Moreover, remarkably similar frequencies of requests for most commonly used tests were identified as they were often ordered together. Such is the case with tests for complete blood count, blood urea nitrogen, creatinine, electrolytes and C-reactive protein, which are requested together in $76 \%$ patients. Different test combinations and sequences used in extended patient management can be identified. Aspartate aminotransferase (AST), alanine aminotransferase (ALT), alkaline phosphatase (ALP), gamma glutamyl transferase (GGT) and bilirubin, historically known as liver function tests, are (with negligible variations) ordered together in 39\% of patients. A total of $45 \%$ of all laboratory requests had, along with other parameters, a troponin $\mathrm{T}(\mathrm{TnT})$ request. Due to the application of the European Society of Cardiology (ESC) guidelines that recommends dynamic determination of TnT (i.e. at patient admission and three hours later), TnT was ordered at two intervals in $9 \%$ of patients, which yields $41 \%$ of TnT requests per patient. The incidence and frequency of ordering laboratory tests shows there is a non-selective overuse of laboratory testing for patients admitted to the emergency department.

\section{WHERE ARE WE HEADING?}

Laboratory overutilization in the emergency department, regardless of the patient's condition, is very complex and requires a multi-systematic approach. The goal of improved utilisation is to make progress in test-ordering behaviour, cost awareness and other factors contributing to laboratory overuse. $(5,7)$ High-quality communication and the maintenance of an open dialogue between laboratory experts and clinicians are key components for both appropriate laboratory utilization and efficient patient care.

As mentioned, laboratory tests in the emergency department should be ordered in the context of the working diagnosis. Laboratory testing based on the determination of pre-test probability (where applicable) can be challenging, but extremely beneficial. Testing in a condition with low pre-test probability can yield false-positive results and cause psychological discomfort and increase workload and expenses, while testing in a high pre-test probability setting can be avoided since the result usually does not alter patient management. (4) The benefits of determining diagnosis likelihoods are numerous and lead to patient-oriented laboratory testing.

Another problem emerges from the fact that the laboratory market is currently overwhelmed with sequential improvements in analytical tests that can detect ever lower concentrations of a specified analyte. Such is the case with immunochemistry assays measuring troponin (both $\mathrm{T}$ and I). Due to its improved analytical and diagnostic sensitivity, as well as tissue specificity, it is inappropriate and useless to request additional biochemical markers of myocardial ischemia and/or injury, such as the activity of creatinine kinase $(\mathrm{CK})$ and its cardiac isoenzyme (CK$\mathrm{MB})$. (8) An elevation of this biomarker serves for emergency physicians as a trigger to refer to a cardiologist, but because of its improved sensitivity, elevated values can be found in patients not experiencing myocardial infarction and can lead to patient misclassification and an amplification of testing. Therefore, every introduction of a new diagnostic test that can have effect on patient management should be discussed with clinicians. In the emergency laboratory of University Hospital Centre Zagreb, a computer-based protocol that eliminates CK from every request where it is coupled with troponin $\mathrm{T}$ was elaborated and notable savings were achieved.

Along with this intervention, a series of other actions have been implemented: the requisition of $\mathrm{D}$-dimers and lipase activ- 
ity exclusively by call, the elimination of estimated sedimentation rate (ESR), total calcium and AST from the request form, as well as the availability of microscopic urinalysis following dipstick analysis only for the paediatric population and urology patients have all contributed to a more efficient laboratory workflow.

Weighing all the points mentioned, the elimination of obsolete tests from the test menu, the setting-up of serum panels according to relevant guidelines and protocols, privilege ordering, computer-based limitation of test availability and the continuous education of clinicians can significantly improve laboratory cost-effectiveness. $(4,5,9,10)$ Some authors (4) claim that a combination of various tools is more effective than a single intervention. In this way, a more patient-oriented approach is achieved, and patients, physicians and laboratories all benefit.

\section{CONCLUSION}

Laboratory testing in the emergency labo- ratory setting should cover the patient's condition and give relevant information for fast and accurate patient management. Excessive and non-evidence based ordering of laboratory tests can be misleading and cause patient discomfort. $(4,5) \mathrm{Co}-$ operation between laboratory experts and clinicians, along with continuous feedback from both sides, is the basis for successful coexistence.

\section{REFERENCES}

1. Topić E. Medicinskobiokemijska dijagnostika hitnih stanja. In: Topić E, Primorac D, Janković S. Medicinskobiokemijska dijagnostika u kliničkoj praksi, Medicinska naklada, Zagreb, 2004, 242-274

2. Rogić D. Laboratorijska dijagnostika hitnih stanja - pretrage uz bolesnika - srčani biljezi. In: Sertić J et al. Klinička kemija i molekularna dijagnostika, Medicinska naklada, Zagreb, 2008, 21-31

3. Ivanović D. Laboratorij i hitna služba. In: Rogić D. Laboratorij i klinika - sučelje o kojem se premalo razgovara, Medicinska naklada, Zagreb, 2009, 15-26

4. Baird G. The laboratory test utilization management toolbox, Biochemia Medica, 2014; 24(2), 223-234

5. Miyakis S, Karamanof G, Liontis M, Mountokalakis TD. Factors contributing to inappropriate ordering of tests in an academic medical department and the effect of an educational feedback strategy, Postgrad Med J, 2006 Dec, 82(974):823-829

6. Klee GG, Westgard JO. Quality Management. In: Tietz Fundamentals of Clinical Chemistry and Molecular Diagnostics, Elsevier, 2015, 90-106

7. Bunting PS, Walraven C. Effect of a controlled feedback intervention on laboratory test ordering by community physicians, Clinical Chemistry, 2004, 50:2, 321-326

8. Casagranda I et al. Proposal for the use in emergency departments of cardiac troponins measured with the latest generation methods in patients with suspected acute coronary syndrome without persistent ST-segment elevation. Clinical Chemistry and Laboratory Medicine, 2013; 51:1727-37

9. Young DS, Sachais BS, Jefferies LC. Laboratory costs in the context of disease, Clinical Chemistry, 2000, 46:7, 967-975

10. Love SA et al. Electronic medical record-based performance improvement project to document and reduce excessive cardiac troponin testing, Clinical Chemistry, 2015, 61:3, 498-504 